\title{
Search for the eta-mesic helium by means of WASA detector at COSY
}

\author{
P. Moskal* \\ M. Smoluchowski Institute of Physics, Jagiellonian University, 30-059 Cracow, Poland, \\ IKP, Forschungszentrum Jülich, D-52425 Jülich, Germany \\ E-mail: p.moskal@uj.edu.pl \\ W. Krzemień \\ M. Smoluchowski Institute of Physics, Jagiellonian University, 30-059 Cracow, Poland, \\ E-mail: wojciech.krzemien@if.uj.edu.pl

\section{Skurzok} \\ M. Smoluchowski Institute of Physics, Jagiellonian University, 30-059 Cracow, Poland, \\ E-mail: magdalena.skurzok@uj.edu.pl
}

\section{for the WASA-at-COSY Collaboration}

The $\eta$-mesic nuclei in which the $\eta$ meson is bound with nucleus via strong interaction was postulated about 25 years ago, however till now no experiment confirmed empirically its existence. The discovery of this new kind of an exotic nuclear matter would be very important for better understanding of the $\eta$ meson structure and its interaction with nucleons. The search for $\eta$-mesic helium is carried out with high statistic and high acceptance with the WASA-at-COSY detection setup and the Cooler Synchrotron COSY. The search is conducted via the measurement of the excitation function for the chosen decay channels of the ${ }^{4} \mathrm{He}-\eta$ system. Until now two reactions $d d \rightarrow\left({ }^{4} \mathrm{He}-\eta\right)_{\text {bound }} \rightarrow{ }^{3} \mathrm{He} p \pi^{-}$and $d d \rightarrow\left({ }^{4} \mathrm{He}-\eta\right)_{\text {bound }} \rightarrow{ }^{3} \mathrm{He} n \pi^{0}$ have been measured with the beam momentum varied continuously around the $\eta$ meson production threshold. This contribution includes description of recently published results of the WASA-at-COSY experiment as well as brief presentation of preliminary results from the analysis of new data sample with 20 times larger statistics.

XV International Conference on Hadron Spectroscopy-Hadron 2013

4-8 November 2013

Nara, Japan

\footnotetext{
${ }^{*}$ Speaker.
} 


\section{Introduction}

The motivation for the search of the mesic nuclei [1] was described in many articles and for the newest results the interested reader is referred to contributions from the recent symposium dedicated to the mesic nuclei e.g. [2-7]. Therefore, here we only briefly recall that the observation of a bound state of meson and nucleus would not only be interesting on its own account, but the determination of properties of $\eta$-mesic or $\eta^{\prime}$-mesic nucleus would be valuable for establishing of the $\eta$ and $\eta^{\prime}$ interactions with nucleons, for studies of the $\mathrm{N}^{*}(1535)$ properties in nuclear matter [8, 9], the properties of the $\eta$ and $\eta^{\prime}$ mesons in the nuclear medium [10-12], for the determination of the flavour singlet components of $\eta$ and $\eta^{\prime}$ mesons [13], and in general for studies of the chiral and axial U(1) symmetry breaking in low energy QCD $[3,9,13]$.

At present, there are ongoing research programs on the $\eta$ and $\eta^{\prime}$-mesic nuclei in many laboratories as e.g.: COSY [14-18], ELSA [19], GSI [21], JINR [22], JPARC [23], LPI [24], and MAMI $[25,26]$. The experimental endeavour to discover the mesic nucleus is supported by the intensive theoretical investigations e.g. [3,7,9,11-13,27-33]. Search for the direct signal from the $\eta$-mesic nucleus are being continued since many years, however so far none of the experiments delivered an unambiguous signal, which could confirm existence of such object. In the case of the production of the $\eta$-mesic helium the established upper limits amount to about $270 \mathrm{nb}$ for the $d p \rightarrow\left({ }^{3} \mathrm{He}-\eta\right)_{\text {bound }} \rightarrow p p p \pi^{-}$reaction [14], about $70 \mathrm{nb}$ for the $d p \rightarrow\left({ }^{3} \mathrm{He}-\eta\right)_{\text {bound }} \rightarrow{ }^{3} \mathrm{He} \pi^{0}$ reaction [14], and about $25 \mathrm{nb}$ for the $d d \rightarrow\left({ }^{4} \mathrm{He}-\eta\right)_{\text {bound }} \rightarrow{ }^{3} \mathrm{He} p \pi^{-}$reaction [16]. The achieved experimental sensitivity is close to the newly predicted values of total cross sections amounting to about $80 \mathrm{nb}$ for the $p d \rightarrow\left({ }^{3} \mathrm{He}-\eta\right)_{\text {bound }} \rightarrow X p \pi^{-}$[4] and $4.5 \mathrm{nb}$ [7] to $30 \mathrm{nb}$ [4] for the $d d \rightarrow\left({ }^{4} \mathrm{He}-\eta\right)_{\text {bound }} \rightarrow X p \pi^{-}$reaction.

There are, however, very encouraging observations indicating indirectly the existence of the $\eta$ mesic helium, which can be summarized as follows: (1) The extremely steep rise of the total cross section for the $p d \rightarrow{ }^{3} \mathrm{He} \eta$ reaction in the very close-to-threshold region followed by a plateau [18, 34] suggesting a pole in the $\eta^{3} \mathrm{He} \rightarrow \eta^{3} \mathrm{He}$ scattering amplitude at the complex excess energy plane $Q$ with $\operatorname{Im}(Q)<0$ [27]. A large enhancement of the total cross section at threshold is observed also for the reactions $d d \rightarrow{ }^{4} \mathrm{He} \eta$ [35] and $p d \rightarrow p d \eta$ [36]; (2) A steep increase of the total cross section for ${ }^{3} \mathrm{He}-\eta$ photo-production at threshold via the $\gamma^{3} \mathrm{He} \rightarrow \eta^{3} \mathrm{He}$ reaction $[6,26]$ shows that the rise of the cross section above threshold is independent of the initial channel and can therefore be assigned to the ${ }^{3} \mathrm{He}-\eta$ interaction; (3) The recent determination of the energy dependence of the tensor analysing power $t_{20}$ by the ANKE collaboration confirmed that the s-wave production amplitude in the $p d \rightarrow{ }^{3} \mathrm{He} \eta$ reaction is fairly energy independent [37] again indicating that the steep threshold enhancement is due to the ${ }^{3} \mathrm{He}-\eta$ interaction; (4) The asymmetry in the angular distribution of the $\eta$ meson emission [18] indicates strong changes of the phase of the s-wave production amplitude with energy, as expected from the occurrence of the bound or virtual $\eta^{3} \mathrm{He}$ state [27]; (5) The evolution with energy of the angular dependence of $\gamma^{3} \mathrm{He} \rightarrow \eta^{3} \mathrm{He}[26]$ is "similar to that of the $p d \rightarrow{ }^{3} \mathrm{He} \eta$ reaction which indicates changing of s-wave amplitude associated with the $\eta^{3} \mathrm{He}$ pole" [4].

In addition, it is worth to stress that an often stated argument that the extracted $\eta N$ scattering length is too low for the $\eta$-helium binding is weakened in view of new theoretical results. Estimates of sub-threshold amplitudes are model dependent and recently Gal et al., has concluded that: "Calculations of $\eta$-nuclear bound states show, in particular, that the $\eta$ - $\mathrm{N}$ scattering length is not a 
useful indicator of whether or not $\eta$ meson bind in nuclei" [5]. Moreover, differences in the value of $\eta N$ scattering lengths obtained in different analyses are at least to some extent explained by the recent observation that the flavour singlet component induces greater binding than the flavour-octet one. Therefore, the $\eta-\eta^{\prime}$ mixing, which is neglected in many of the former analyses, increases the $\eta$-nucleon scattering length relative to the pure octet $\eta$ by a factor of about 2 [3].

The binding energies of $\eta$ and $\eta^{\prime}$ in nuclear medium are sensitive to the non-perturbative glue $[3,13]$ and due to the $\mathrm{U}_{\mathrm{A}}(1)$ anomaly effect, a relatively large mass reduction of $\eta^{\prime}$ meson is expected in medium [38] which may indicate the existence of the $\eta^{\prime}$-mesic nucleus [38] as predicted in [29]. Therefore, in spite of the indications that $\eta^{\prime}$-nucleon interactions is smaller than $\eta$-nucleon [39], recently there are vigourous theoretical investigations $[3,9,28,30,40]$ and preparations of experiments for the $\eta^{\prime}$-mesic nucleus [20,21]. These experiments are motivated also by the recent photoproduction measurements of CBELSA/TAPS [19] showing that the real part of the $\eta^{\prime}$-nucleus optical potential is larger than its imaginary part.

\section{Status of the search for $\eta$-mesic helium with WASA-at-COSY}

The WASA detector [41] at COSY gives unique possibilities to conduct studies of the hadronic production of He- $\eta$ system with the continuous change of the beam momentum and the exclusive measurement of all ejectiles. Two experiments dedicated to the search of $\eta$-mesic helium were conducted up to now using the WASA-at-COSY detector. Both were focused on the bound state decay into the ${ }^{3} \mathrm{He}$ and a nucleon-pion pair $[15,42]$

The first experiment was performed in June 2008 by measuring the excitation function of the $d d \rightarrow{ }^{3} \mathrm{He} p \pi^{-}$reaction near the $\eta$ meson production threshold [16]. During the experimental run the momentum of the deuteron beam was varied continuously within each acceleration cycle from $2.185 \mathrm{GeV} / \mathrm{c}$ to $2.400 \mathrm{GeV} / \mathrm{c}$, crossing the kinematic threshold for $\eta$ production in the $d d \rightarrow{ }^{4} \mathrm{He} \eta$ reaction at $2.336 \mathrm{GeV} / \mathrm{c}$. This range of beam momenta corresponds to a variation of the ${ }^{4} \mathrm{He}-\eta$ excess energy from $-51.4 \mathrm{MeV}$ to $22 \mathrm{MeV}$. The data show no statistically significant signal of the ${ }^{4} \mathrm{He}-\eta$ bound state in the excitation function [16], and the determined upper limit for the crosssection for the bound state formation and decay in the process $d d \rightarrow\left({ }^{4} \mathrm{He}-\eta\right)_{\text {bound }} \rightarrow{ }^{3} \mathrm{He} p \pi^{-}$varies from $20 \mathrm{nb}$ to $27 \mathrm{nb}$ at $90 \% \mathrm{CL}$ [16]. The established upper limits depend mainly on the width of the bound state and only slightly on the binding energy.

In the second experiment, conducted in November 2010, we increased the statistics with respect to the previous results [16] by a factor of about 20. During the second experimental campaign two reactions were measured simultaneously: $d d \rightarrow\left({ }^{4} \mathrm{He}-\eta\right)_{\text {bound }} \rightarrow{ }^{3} \mathrm{He} p \pi^{-}$and $d d \rightarrow\left({ }^{4} \mathrm{He}-\right.$ $\eta)_{\text {bound }} \rightarrow{ }^{3} \mathrm{Hen} \pi^{0} \rightarrow{ }^{3} \mathrm{Hen} \gamma \gamma$ [15]. The deuteron beam momentum was varied continuously within each acceleration cycle in the momentum range corresponding to the variation of the ${ }^{4} \mathrm{He}-\eta$ excess energy from $-70 \mathrm{MeV}$ to $30 \mathrm{MeV}$. The analysis of the data is still in progress. Preliminary spectra of the excitation function have been presented in [42]. The shape of the excitation functions determined for the range of small ${ }^{3} \mathrm{He}$ momenum, when a signal from the bound state is expected, differes from the shape of the excitation function for the range with larger ${ }^{3} \mathrm{He}$ momenum. This is a promising result, but its final interpretation requires detailed simulations in order to understand the background contributions to the observed excitation functions. 
Anyhow, already now we can conclude that a collected data are of a very good quality and that we have achieved a sensitivity of the cross section of the order of few nb for the bound state production in $d d \rightarrow\left({ }^{4} \mathrm{He}-\eta\right)_{\text {bound }} \rightarrow{ }^{3} \mathrm{He} n \pi^{0}$ and $d d \rightarrow\left({ }^{4} \mathrm{He}-\eta\right)_{\text {bound }} \rightarrow{ }^{3} \mathrm{He} p \pi^{-}$reactions. This is in the range of cross section value of $4.5 \mathrm{nb}$ predicted in [7].

\section{Perspectives}

For possible future investigations of the $\eta$-mesic helium an interesting possibility of non$N \pi$ decays of the mesic-helium was pointed out by Wycech [7] and Wilkin [4]. In this case the reaction may proceed e.g. as follows: $p d \rightarrow\left({ }^{3} \mathrm{He}-\eta\right)_{\text {bound }} \rightarrow p p n$ or $p d \rightarrow\left({ }^{3} \mathrm{He}-\eta\right)_{\text {bound }} \rightarrow p d$. Such processes could be due to the absorption of $\eta$-meson via e.g. $\eta d \rightarrow p n$ reaction. However, Wilkin [4] estimated in the first approximation that the two-nucleon absorption constitutes at most $5 \%$ of the total decay rate, and in addition these channels are buried in a large background.

Another possible mechanism of the decay of the bound state is the decay of the $\eta$ meson when it is still orbiting around the nucleus. This seems to be promising experimentally due to the very low background. Using arguments given in [4], as a very rough approximation we may estimate the cross sections for the processes: $p d \rightarrow\left({ }^{3} \mathrm{He}-\eta\right)_{\text {bound }} \rightarrow{ }^{3} \mathrm{He} 2 \gamma$, and $p d \rightarrow\left({ }^{3} \mathrm{He}-\eta\right)_{\text {bound }} \rightarrow{ }^{3} \mathrm{He} 6 \gamma$ to be about $0.4 \mathrm{nb}$. This value can be estimated taking into account that the total width of the $\eta$ meson is about $1.3 \mathrm{keV}$, the width of the $\left({ }^{3} \mathrm{He}-\eta\right)$ is less than about $500 \mathrm{keV}$, and the $2 \gamma$ and $6 \gamma$ branching ratios amounts to about $39 \%$ and $33 \%$, respectively [4].

\section{Acknowledgements}

We acknowledge support by the Foundation for Polish Science (MPD programme), by the Polish National Science Center through grant: No. 2011/01/B/ST2/00431, and by the FFE grants of the Research Center Jülich.

\section{References}

[1] Q. Haider, L. C. Liu, Phys. Lett. B172, 257 (1986).

[2] International Symposium on Mesic Nuclei, Cracow 2013, http://koza.if.uj.edu.pl/mesic-nuclei-2013/

[3] S. D. Bass, A. W. Thomas, e-Print: arXiv:1311.7248, Acta Phys. Pol. $\mathbf{B 4 5}$ (2014), in print.

[4] C. Wilkin, Acta Phys. Pol. $\mathbf{B 4 5}$ (2014), in print.

[5] A. Gal et al., Acta Phys. Pol. $\mathbf{B 4 5}$ (2014), in print.

[6] B. Krusche, Acta Phys. Pol. $\mathbf{B 4 5}$ (2014), in print.

[7] S. Wycech, W. Krzemien, e-Print: arXiv:1401.0747; Acta Phys. Pol. B45 (2014), in print.

[8] D. Jido, H. Nagahiro, S. Hirenzaki, Phys. Rev. C66, 045202 (2002).

[9] S. Hirenzaki et al., Acta Phys. Polon. B41, 2211 (2010).

[10] T. Inoue, E. Oset, Nucl. Phys. A710, 354 (2002).

[11] E. Friedman, A. Gal, J. Mares, Phys. Lett. B725, 334 (2013). 
[12] A. M. Green, S. Wycech, Phys. Rev. C71, 014001 (2005).

[13] S. D. Bass, A. W. Thomas, Phys. Lett. B634, 368 (2006).

[14] P. Moskal, J. Smyrski, Acta Phys. Pol. B41, 2281 (2010).

[15] M. Skurzok, P. Moskal, W. Krzemien, Prog. Part. Nucl. Phys. 67, 445 (2012).

[16] P. Adlarson et al., Phys. Rev. C87, 035204 (2013).

[17] A. Budzanowski et al., Phys. Rev. C79 012201 (2009).

[18] J. Smyrski et al., Phys. Lett. B649, 258 (2007); T. Mersmann et al., Phys. Rev. Lett. 98, 242301 (2007).

[19] M. Nanova et al., Phys. Lett. B710, 600 (2012); M. Nanova et al., Phys. Lett. B727, 417 (2013).

[20] V. Metag et al., approved ELSA/03-20120BGO-OD.

[21] Y. K. Tanaka et al., Few Body Syst. 541263 (2013).

[22] S.V. Afanasiev, Phys. Part. Nucl. Lett. 81073 (2011).

[23] H. Fujioka, Acta Phys. Polon. B41, 2261 (2010).

[24] Baskov V. A. et al., PoS Baldin-ISHEPP-XXI 102 (2012).

[25] B. Krusche et al., J. Phys. Conf. Ser., 349, 012003 (2012).

[26] F. Pheron et al., Phys. Lett. B709, 21 (2012).

[27] C. Wilkin, Phys. Lett. B654, 92 (2007).

[28] H. Nagahiro et al., Phys. Rev. C87 045201 (2013).

[29] H. Nagahiro, S. Hirenzaki, Phys. Rev. Lett. 94232503 (2005).

[30] H. Nagahiro et al., Phys. Lett. B709 (2012) 87.

[31] A. Cieply et al., e-Print: arXiv:1312.1547.

[32] N. G. Kelkar et al., Rept. Prog. Phys. 76, 066301 (2013).

[33] S. D. Bass, A. W. Thomas, Acta Phys. Polon. B41 2239 (2010); arXiv:1007.0629 [hep-ph].

[34] J. Berger et al., Phys. Rev. Lett. 61, 919 (1988); B. Mayer et al., Phys. Rev. C53, 2068 (1996); M. Betigeri et al., Phys. Lett. B472, 267 (2000); H. H. Adam et al., Phys. Rev. C75, 014004 (2007); J. Smyrski et al., Acta Phys. Slov. 56213 (2006).

[35] R. Frascaria et al., Phys. Rev. C50, 537 (1994); N. Willis et al., Phys. Lett. B406, 14 (1997); A. Wronska et al., Eur. Phys. J. A26, 421 (2005); A. Budzanowski et al., Nucl. Phys. A821, 193 (2009).

[36] F. Hibou et al., Eur. Phys. J. A7, 537 (2000); R. Bilger et al., Phys. Rev. C69, 014003 (2004); C. Piskor-Ignatowicz et al., Int. J. Mod. Phys. A22, 528 (2007).

[37] A. Khoukaz et al., Acta Phys. Pol. B45 (2014), in print.

[38] S. Hirenzaki, H. Nagahiro, Acta Phys. Pol. B45 (2014), in print.

[39] P. Moskal et al., Phys. Lett. B482, 356 (2000).

[40] H. Nagahiro, D. Jido, S. Hirenzaki, Phys. Rev. $\mathbf{C 8 0} 025205$ (2009).

[41] H. H. Adam et al., e-Print: nucl-ex/0411038.

[42] W. Krzemien, P. Moskal, M. Skurzok, e-Print: arXiv:1401.6644. 